\title{
A 1.5 MICRO-LITER MICROBIAL FUEL CELL ARRAY FOR RAPID SCREENING OF EXOELECTROGENIC BACTERIA
}

\author{
S. Choi ${ }^{*}$, S. Mukherjee ${ }^{1}, S . S u^{2}$, W. Panmanee ${ }^{3}$, R.T. Irvin ${ }^{2,4}$, and D. J. Hassett ${ }^{3,4,5}$ \\ ${ }^{1}$ School of Electronic and Computing Systems, University of Cincinnati, Ohio, USA \\ ${ }^{2}$ Depaertment of Microbiology and Immunology, University of Alberta, CA, \\ ${ }^{3}$ Department of Molecular Genetics, Biochemistry \& Microbiology, University of Cincinnati, Ohio, USA, \\ ${ }^{4}$ Pilus Energy LLC, Cincinnati, Ohio, USA, ${ }^{5}$ Cincinnati VA Hospital, Cincinnati, OH USA
}

\begin{abstract}
We report an array of six MEMS microbial fuel cells (MFCs), a compact and reliable platform for rapid screening of exoelectrogenic bacteria. The presented device contains vertically stacked $1.5 \mu \mathrm{L}$ anode/cathode chambers separated by a proton exchange membrane (PEM), and represents the smallest MEMS MFC array. Within just five hours, we successfully determined the electricity generation capacity of two known bacterial electrogens and another metabolically more voracious organism with 4 isogenic mutants of this strain constructed with the hypothesis that such mutations could alter their electrogenic properties. In addition, the percent deviation of all six MFC units was less than $1.4 \%$ from their open circuit voltages recorded, which is far less than that of mL-sized MFC arrays and even MEMS MFC arrays.
\end{abstract}

\section{INTRODUCTION}

Driven by increasing concerns over the energy-climate crisis and environment pollution, microbial fuel cells (MFCs) have been a major focus for renewable energy production [1]. MFCs are powered by live microorganisms to efficiently catalyze degradation of a broad range of organic substrates under natural conditions, and, as such, offer clean and sustainable features [2]. Thus far, only a limited number of bacteria species and their optimal growth conditions have been studied for use in various MFCs. This reveals a significant deficiency of the essential knowledge needed to ascertain which bacterial species or consortia may be best suited for generating optimal power density in MFCs [3]. This deficiency is largely caused by limitations in current screening methods based on larger scale two-bottle MFCs that require longer start-up times (from days to weeks), significant space and materials (hundreds of milliliters to liters), and labor-intensive control for MFC experiments, either in series or in parallel circuits [4]. This limitation has motivated efforts to miniaturize MFC arrays, such that the effective chamber volumes are reduced to the microliter scale in a well-controlled manner. However, recently proposed 9-well pipette tip-based miniature MFC array [3] and 24-well MEMS MFC array [5, 6] still has several limitations; (1) large sized anodic chamber volume $(>400 \mu \mathrm{L})$, and (2) high percent deviation in each MFC unit $(>8 \%)$. Here, we developed an ultra-small $(1.5$ $\mu \mathrm{L})$, reproducible, and practical parallel analyses platform for rapid screening of exoelectrogenic bacteria. Normally, miniaturizing MFCs into a few micro-scale levels is exceptionally challenging because the devices become vulnerable to the $\mathrm{O}_{2}$ invasion into the anode chamber, leading to a significant increase in the start-up time and decrease in current generation [7]. Typically, microorganisms consume $\mathrm{O}_{2}$ immediately and mitigate abiotic $\mathrm{O}_{2}$ reduction on the anode; this $\mathrm{O}_{2}$-scavenging reaction occurs in macro-sized MFCs having high biomass concentration in an anode chamber. However, the micro-sized MFC has a small anode chamber volume, and the small-area anode had a small number of $\mathrm{O}_{2}$-utilizing microorganisms [7]. Therefore, in order to minimize $\mathrm{O}_{2}$ intrusion in such a small MFC array, we used $254 \mu$ m-thick PTEE (polytetrafluoroethylene) gaskets (five orders of oxygen permeability

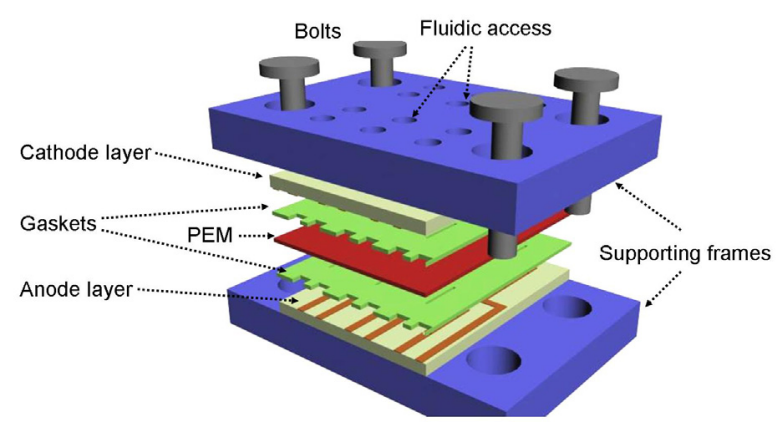

Figure 1: Schematic of the 6-well MEMS MFC array system.
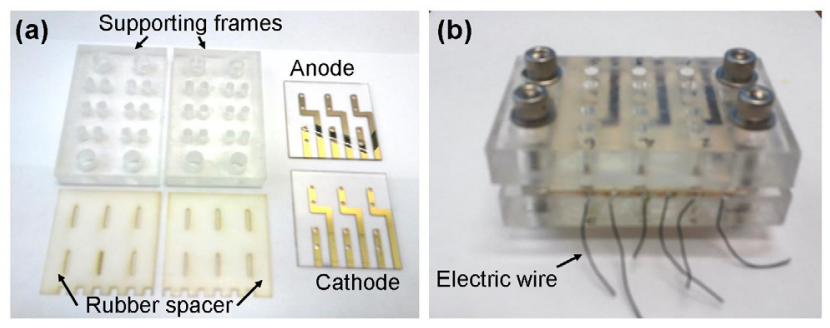

Figure 2: (a) Individual layers of the MFC array and (b) fully assembled MFC array.

lower than that of other polymer gaskets) and PEEK (Polyetheretherketone) polymer tubings (fifty times lower magnitude than that of conventional fluorinated ethylene propylene tubings) [8]. Finally, we applied this technique for studies of selected hypothesis-driven genes that could alter power density in $P$. aeruginosa isogenic mutants. These efforts demonstrated that spatially distinct wells of the MFC array displayed highly comparable performance characteristics and identified which of the hypothesis-driven, selected genes in $P$. aeruginosa triggered higher power density. The hyperpiliated pilT mutant of $P$. aeruginosa displayed the highest current generation, 2-fold higher than the well known electrogenic bacterium, Shewanella oneidensis.

\section{MATERIALS AND METHODS}

\section{Device fabrication and assembly}

A schematic of the MFC array is shown in Fig. 1. The MFC array consisted of five functional layers; an anode electrode layer (Cr/Au on PMMA (polymethyl methacrylate)), an anode chamber layer (gasket), a PEM (proton exchange membrane), a cathode chamber layer (gasket), and a cathode electrode layer $(\mathrm{Cr} / \mathrm{Au}$ on PMMA). The $1.5 \mu \mathrm{L}$ anode/cathode chamber was defined by a 254 $\mu \mathrm{m}$-thick gasket; the exposed electrode area per cell is $6 \mathrm{~mm}^{2}$ (Fig. 2a). The array was assembled by the two supporting frames with 
bolts and nuts (Fig. 2b). Each layer except for the PEM was micro-patterned by using laser micromachining. First, we cut two 5 x $5 \mathrm{~cm}$ PMMA chips with the laser and drilled 12 holes on each chip for inlets/outlets. The chips were then coated with $\mathrm{Cr} / \mathrm{Au}(20$ $\mathrm{nm} / 200 \mathrm{~nm}$ ) by an electron-beam evaporator and patterned to define the electrodes using a lift-off process. The $254 \mu \mathrm{m}$-thick PTEE rubber gaskets were micro-patterned to define the anode/cathode chambers. All the layers were manually stacked in sequence while carefully aligning the tubing holes for the microfluidic channels. For fluidic access, 24 sterile PEEK polymer tubings were plugged into the holes to form twelve independent routes for anolyte/catholyte access. The individual microfluidic access path is very important to provide independent and reliable results for each MFC unit.

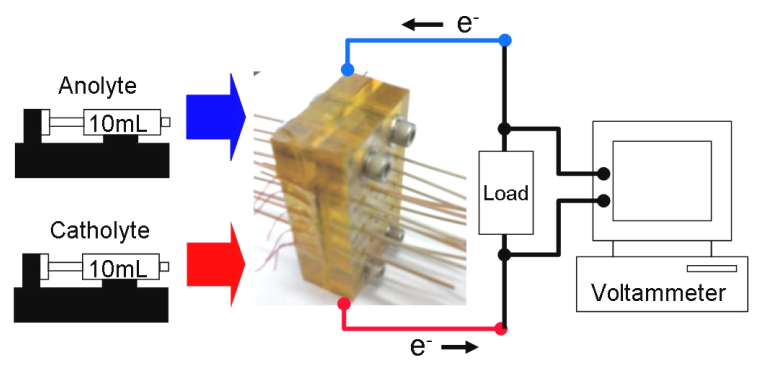

[ Solution Injection ] [ [ LL-MFC ] [ Data Acquisition ]

Figure 3: Schematic of test setup for monitoring the MEMS MFC array. The array has 6 microfluidically-controlled micro-sized MFCs integrated on a single chip. All six MFCs are connected to 150- $\Omega$ external loads and output voltages are recorded every 10 $\min$.

\section{Inoculum}

For the selection of hyper-electrogenic strains, we used Luria broth in the anodic chamber, and, for the sake of convenience, aerobic $50 \mathrm{mM}$ potassium ferricyanide in the cathode buffered with $100 \mathrm{mM}$ potassium phosphate, $\mathrm{pH}$ 7.0. Six microorganisms were tested; Wild-type $S$. oneidensis MR1, Wild-type $P$. aeruginosa $P A O 1$ and isogenic nirS, lasl, bdlA and pilT mutants, respectively. $P$. aeruginosa $P A O 1$ mutants were generated using classical allelic replacement techniques with gentamicin or tetracycline cassettes inserted with selected genes followed by sucrose counter-selection to resolves merodiploids as described by Hoang et al. [9].

\section{Measurement setup}

We measured the potentials between the anodes and cathodes using a data acquisition system (CompactRIO System, National Instrument) and recorded them every $10 \mathrm{~min}$. via LabVIEW. An external resistor $(150 \Omega)$, connected between the electrodes of each MFC unit, closed the circuit. We calculated current through the resistor via Ohm's law. Current density was normalized to the anode area $\left(6 \mathrm{~mm}^{2}\right)$ or anode chamber volume $(1.5 \mu \mathrm{L})$. We droved anolyte and catholyte to fill the anode/cathode chambers with syringe pumps, and supplied the solutions continuously at a very low flow rate, under $20 \mu \mathrm{L} /$ hour.

\section{RESULTS AND DISCUSSION}

Open circuit voltages

We first compared the Open Circuit Voltages (OCV; cathode potential minus anode potential with an infinite load) in the

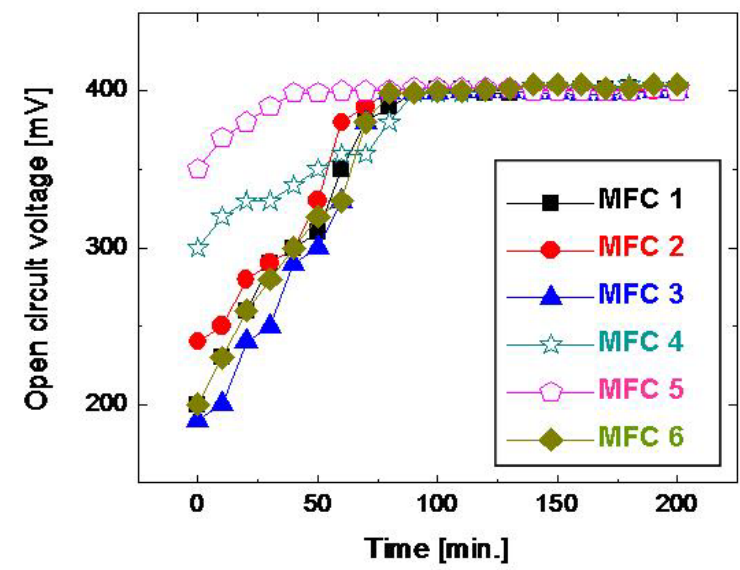

Figure 4: Open circuit voltages $(\mathrm{OCV})$ of the $\mathrm{MFC}$ units in the array.

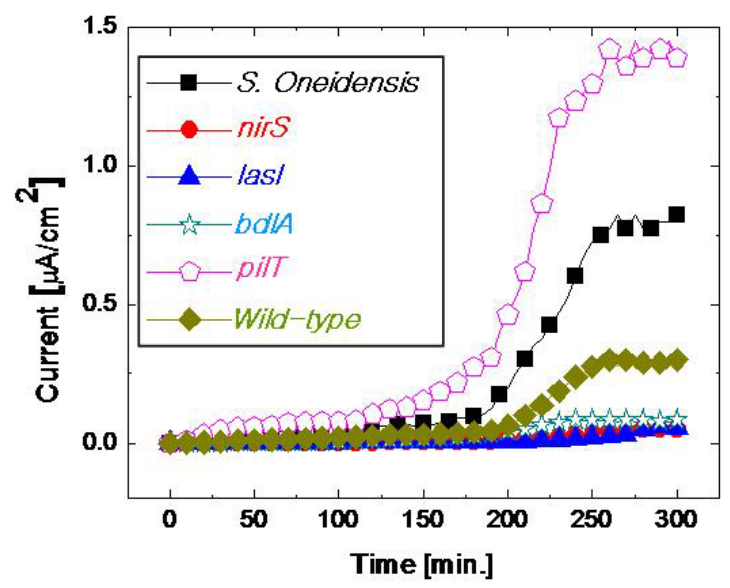

Figure 5: Currents produced from six MFC units, in which different microorganisms are inoculated, respectively. pilT mutant of $P$. aeruginosa (pentagons) produced the highest current.

continuous-mode MFC units. No bacteria were inoculated in the anode chambers for these experiments, in which the cathode potentials were controlled by ferricyanide at about $300 \mathrm{mV}$. The MFC array was built airtight and all solutions were sparged with $\mathrm{N}_{2}$ before injection. As shown in Fig. 4, the OCVs of the six MFCs increased and reached a value of approximately $402 \mathrm{mV}$ with less than $1.4 \%$ variation, which is far less than that of $\mathrm{mL}$-sized MFC array $(25 \%)$ and even other MEMS MFC arrays ( $>8 \%)$ [2, 10, 11]. We believe that this low percent deviation is attributed to the (1) significantly reduced chamber volume in a well-controlled manner and (2) more effective oxygen-tight interface. As the size of the MFCs becomes smaller, it has been observed that the background current decreased drastically in the $\mathrm{nA}$ range [12]. Since we used a $\mathrm{mm}^{2}$ scale anode area, the background current was almost negligible and became stable. Also, we recently reported that $\mathrm{O}_{2}$ penetrating into the anode chamber abiotically reacted with the anode since the anode potential was negative enough $(-0.3$ to $-0.1 \mathrm{~V}$ versus $S H E)$ to reduce $\mathrm{O}_{2}$ into $\mathrm{H}_{2} \mathrm{O}$ or low $\mu \mathrm{M}$ levels of $\mathrm{H}_{2} \mathrm{O}_{2}$. This reduction will create a negative current that competes with the positive current produced by the microorganisms. To date, prior micro-sized MFC arrays were not able to minimize the $\mathrm{O}_{2}$ invasion into the anode 
Table 1: Micro-sized MFC arrays

\begin{tabular}{|c|c|c|c|c|c|}
\hline & (1) Biffinger et al. 2009 [3] & (2) Hou et al. 2009 [11] & (3) Hou et al. 2011a [5] & (4) Hou et al. 2011b [6] & (5) This work \\
\hline \# of MFC units & 9 & 24 & 24 & 24 & 6 \\
\hline $\begin{array}{l}\text { \# of actual bacterial species to be } \\
\text { screened }\end{array}$ & 9 & 24 & 24 & 6 & 6 \\
\hline Bacteria & Shewanella sp. & Shewanella $s p$. & $\begin{array}{l}\text { Shewanella \& } \\
\text { Arthrobacter sp. }\end{array}$ & Shewanella $s p$. & $\begin{array}{c}\text { Shewanella \& } \\
\text { Pseudomonas sp. }\end{array}$ \\
\hline Operation/fluidic access & Batch mode / no access & Batch mode / no access & Batch mode / no access & $\begin{array}{l}\text { Continuous mode / } \\
4 \text { common access }\end{array}$ & $\begin{array}{l}\text { Continuous mode / } \\
6 \text { independent access }\end{array}$ \\
\hline Sustainability & $<15$ hours & $<17$ hours & NA & $>$ Several days & $>$ Several days \\
\hline Anode/cathode material & Graphite felt/Carbon paper & Gold/Carbon Cloth & Carbon Cloth /Carbon Cloth & Gold/Carbon paper & Gold/Gold \\
\hline Anode area & $1.1 \mathrm{~cm}^{2}$ & $0.38 \mathrm{~cm}^{2}$ & $0.38 \mathrm{~cm}^{2}$ & $0.38 \mathrm{~cm}^{2}$ & $0.06 \mathrm{~cm}^{2}$ \\
\hline Anode volume & $500 \mu \mathrm{L}$ & $600 \mu \mathrm{L}$ & $600 \mu \mathrm{L}$ & $400 \mu \mathrm{L}$ & $1.5 \mu \mathrm{L}$ \\
\hline Membrane & Nafion 117 & Nafion 117 & Nafion 117 & Nafion 117 & Nafion 117 \\
\hline Catholyte & Potassium ferricyanide & Potassium ferricyanide & Air & Potassium ferricyanide & Potassium ferricyanide \\
\hline Start-up & $<2$ hours & $<2$ hours & $<20$ hours & NA & $<2$ hours \\
\hline Deviation between array cells & $8 \%$ & $6 \sim 14 \%$ & $16.4 \sim 22 \%$ & NA & $1.4 \%$ \\
\hline Current density & $\sim 2.5 \mu \mathrm{A} / \mathrm{cm}^{2}$ & $\sim 0.5 \mu \mathrm{A} / \mathrm{cm}^{2}$ & $\sim 0.2 \mu \mathrm{A} / \mathrm{cm}^{2}$ & NA & $\sim 1 \mu \mathrm{A} / \mathrm{cm}^{2}$ \\
\hline
\end{tabular}

chamber since they used PDMS or adhesive as a gasket, both of which have high $\mathrm{O}_{2}$ permeability [7]. Eventually, they could have different amounts of $\mathrm{O}_{2}$ dissolved in the chambers, leading to significant deviations in each MFC unit. Therefore, $\mathrm{O}_{2}$ leakage in the micro-scale MFC array was the dominant factor for their unreliable and irreproducible properties while high percent deviation in the conventional macro-scale MFCs was mainly due to the large background noise and the large variations in the growth, evolution, and metabolism of the consortium of microbes in the uncontrollable macro-scale environmental surroundings. Here, in order to have low percent deviation between MFC units in the array, we reduced the chamber volume to $1.5 \mu \mathrm{L}$, which is the smallest chamber among all the reported MFC arrays. In addition, we minimized $\mathrm{O}_{2}$ intrusion by using $254 \mu \mathrm{m}$-thick PTEE gaskets and PEEK polymer tubings [8].

\section{Current generation and start-up time}

Six bacterial cultures (one $S$. oneidensis and five different $P$. aeruginosa strains) and potassium ferricyanide were continuously supplied to the anode and cathode chambers, respectively, with flow driven by rate-controlled syringe pumps. Normally, the MFCs are operated under batch mode for better accumulation and acclimation of bacteria for optimal biofilm formation on their anode surfaces [7, 12], a trait necessary for mediator-less current generation. Once current generations are observed after bacterial inoculation, solutions are supplied under continuous mode. However, as the size of the MFC is scaled towards the micro regime, it is difficult to notice the biofilm formation by monitoring its current generation because it becomes extremely sensitive to even minute $\mathrm{O}_{2}$ leakage and interrupt bacteria from attaching the surface for anode-respiration, dramatically decreasing current generation and increasing start-up time [7, 8]. That is why we continuously flowed all culture solutions even for biofilm formation on the anode but at a very low flow rate $(<20 \mu \mathrm{L} /$ hour $)$. This continuous operation helped keep a high flow by flushing out $\mathrm{O}_{2}$ that had diffused into the anode and support better anode-electron transfer for the bacteria, ultimately decreasing start-up phase and increasing current density. Reducing oxygen diffusion is the key factor to increasing the performance of micro-sized MFCs because $\mathrm{O}_{2}$ diffusion into the anode chamber could compete with extracellular electron transfer to the anode and, therefore, decrease coulombic efficiency and current generation. For example, it has been reported that Geobacter $s p$. can grow with $\mathrm{O}_{2}$ as a terminal electron acceptor, and this diverts electrons away from the anode, resulting in lower current density [14]. Maximizing the performance of an individual MFC unit on the array is critical to the successful development of a sensitive MFC array. All six MFCs started to generate current about $100 \mathrm{~min}$. after inoculum injection (Fig. 5). Then, the current gradually increased and reached a peak value in 5 hours. This demonstrates the capability of our MEMS MFC array for a fast analysis of exoelectrogenic bacteria compared to that of most mL-scale MFCs (several days) and it is comparable to that of other MEMS MFC arrays. We successfully determined the electrogenic capacity from 4 mutants of $P$. aeruginosa with high sensitivity, which has never been investigated. The hyperpiliated pilT mutant of $P$. aeruginosa displayed the highest current generation, 2-fold higher than that of wild-type $S$. oneidensis. This finding is quite interesting because reducing twitching motility could increase the current generation. This work validated the utility of our MFC array by studying how strategic genetic modifications impact the electrochemical activity of $P$. aeruginosa strains.

\section{Comparison to other micro-sized MFC arrays}

Table 1 summarizes specifications of prior micro-sized MFC array work and compares our MEMS MFC array with them. Initial MFC arrays were operated under batch mode without fluidic access. Even the first MFC array [3] was completely exposed to air and was not able to remain active in anaerobic environments, eliminating its ability to study many other bacteria strains that expire when exposed to aerobic environments for extended periods of time (Geobacter sp., Clostridium sp., etc.). The second and third MFC arrays [5,6,11] were also exposed to some air since the device required solution loading into each chamber between manual assembly steps for the array, leading to unreliable/irreproducible results. Another common concern with these three MFC arrays can cause contamination from unit to unit during operation or fabrication. Moreover, lack of fluidic access to each MFC unit hampers long-term analysis abilities ( $<17$ hours). In this sense, continuous mode is ideal for the MFC array. Very recently, Hou et al., for the first time, reported a microfluidically-controlled MFC array [6]. To 
simplify the intricate microfluidic accesses, they used 4 common fluidic access to the anode chamber and one common access to the cathode chamber, demonstrating long term screening studies of electrogens. However, they have not presented enough valuable data we can compare to with other MFC arrays. Moreover, the device does not provide independent access of the 24 spatially distinct MFC units and thus, in actuality, only 6 different microbial species can be screened. Our MFC array significantly reduced the chamber volume and anode area, leading to (1) low background current, (2) very low deviation between MFC units, and (3) short start-up time despite the fact that the reduced chamber becomes more sensitive to oxygen invasion and diverts electrons away from the anode.

Gold has been identified as a potential material for micro-sized MFC anode development. Gold is biocompatible, highly conductive, and is compatible with conventional microfabrication modalities for the development of a micro-sized MFC platform. However, our preliminary results with Geobacter $s p$. and other previous studies with Shewanella $s p$. suggested that bare gold is a poor electrode material for the anode of MFCs because gold does not contain functional groups, such as redox-capable quinones, a natural electron acceptor for anaerobic respiration [7, 15]. As shown in Table 1, the MFC array using graphite felt as an anode generated more than 5 times larger current density than that using gold. We believe that the most effective approach for developing efficient micro-sized MFC anodes will be to chemically modify the gold surface for better electronic coupling between gold electrodes and bacteria along with maintaining compatibility to MEMS techniques. For example, current production with $S$. putrefaciens increased 100 -fold when the gold surface was coated with a SAM of 11-mercapto-undecanoic acid [16]. This was due to bacterial cytochromes conducting the final electron transfer from the bacteria and the carboxylic acid termini of SAM-modified electrodes can accommodate cytochromes on an electrode surface through strong hydrogen bonding with the peptide bonds within the protein backbone. Another option for the micro-sized MFC anode might be alternative electrode materials or non-conventional nanoscale electrodes such as nanowires, nanotubes, or graphenes, meeting micro-/nano-fabrication process requirements.

\section{CONCLUSION}

In this work, we developed a $1.5 \mu \mathrm{L} \mathrm{MFC}$ array that allows for reliable and sensitive bacterial screening within just five hours. The OCVs of 6 spatially distinct MFC units on the array differed by less than $1.4 \%$. Ultimately, we were able to validate the utility of our MFC array by studying selected hypothesis-driven genes in $P$. aeruginosa. We expect that this work highlights the potential realization of a practical tool for efficient high-throughput bacterial screening and fundamental MFC understanding, which may further improve power extraction in MFCs. Our future work is directed toward the development of much higher throughput micro-sized MFC arrays with independent fluidic accesses.

\section{ACKNOWLEDGEMENT}

We thank Prof. Jason Heikenfeld for stimulating discussions and offering part of the fabrication facilities. We would also like to thank Ohio Center for Microfluidic Innovation (OCMI) at the University of Cincinnati for supporting this work.

\section{REFERENCES}

[1] Y. Yang, G. Sun, and M. Xu, "Microbial fuel cells come of age," J. Chem. Technol. Biotechnol. 86, 625 (2010)

[2] L. Logan, and J.M. Regan, "Microbial fuel cells - challenges and applications," Environ. Sci. Tehcnol., 40, 5172 (2006)
[3] J. Biffinger, M. Ribbens, B. Ringeisen, J. Pietron, S. Finkel, and K. Nealson, "Characterization of electrochemically active bacteria utilizing a high-throughput voltage-based screening assay," Biotechnology and Bioengineering, 102, 436 (2009)

[4] X. Cao, X. Huang, X. Zhang, P. Liang, and M. Fan, "A mini-microbial fuel cell for voltage testing of exoelectrogenic bacteria," Front. Environ. Sci. Engin. China. 3, 307 (2009)

[5] H. Hou, L. Li, P. de Figueiredo, and A. Han, "Air-cathode microbial fuel cell array: A device for identifying and characterizing electrochemically active microbes," Biosensors and Bioeletronics, 26, 2680 (2011)

[6] H. Hou, L. Li, P. de Figueiredo, and A. Han, "Microfluidic microbial fuel cell array for multiplexed long-term parallel analysis of microbial activities, "Proceedings of 15 th International Conference on Miniaturized Systems for Chemistry and Life Sciences ( $\mu$-TAS) 2011, Seattle, USA, (2011), pp. 1424-1426.

[7] S. Choi, H.-S. Lee, Y. Yang, P. Parameswaran, C.I. Torres, B.E. Rittmann and J. Chae, "A $\mu \mathrm{L}$-scale Micromachined Microbial Fuel Cell Having High Power Density," Lab on a Chip, 11, 1110 (2011)

[8] S. Choi, J. Chae, " $\mu L$-Scale microbial fuel cell with optimal power generation and biofilm formation," Proceedings of $25^{\text {nd }}$ IEEE International Conference on Micro Electro Mechanical Systems (MEMS) 2012, Paris, France, (2012), pp.43- 46.

[9] T.T. Hoang, A.J. Kutchma, A. Becher, and H.P. Schweizer, "Integration-proficient plasmids for Pseudomonas aeruginosa: site-specific integration and use for engineering of reporter and expression strains," Plasmid, 43, 59 (2000)

[10] P. Aelterman, K. Rabaey, H.T. Pham, N. Boon, and W. Verstraete, "Continuous electricity generation at high voltages and currents using stacked microbial fuel cells," Environ. Sci. Technol., 40, 3388, (2006)

[11] H. Hou, L. Li, Y. Cho, P. de Figueiredo, and A. Han, "Microfabricated Microbial Fuel Cell Arrays Reveal Electrochemically Active Microbes," PLOS one, 4, e6570, (2009)

[12] F. Qian, M. Baum, Q. Gu, and D. E. Morse, "A $1.5 \mu l$ microbial fuel cell for on-chip bioelectricity generation," Lab on a Chip, 9 , 3076 (2009)

[13] Z. He, J. Kan, Y. Wang, Y. Huang, F. Mansfeld, and K.H. Nealson, "Electricity production coupled to ammonium in a microbial fuel cell," Environ. Sci. Technol. 43, 3391 (2009)

[14] W.C. Lin, M.V. Coppi, D.R. Lovley, "Geobacter sulfurreducens Can Grow with Oxygen as a Terminal Electron Acceptor," Appl. Environ. Microbiol. 70, 2525 (2004)

[15] H. Richter, K. McCarthy, K.P. Nevin, J.P. Johnson, V.M. Rotello, and D.R. Lovley, "Electricity generation by Geobacter Sulfurreducens attached to gold electrodes," Langmuir, 24, 4376 (2008)

[16] S.R. Crittenden, C.J. Sund, and J.J. Sumner, "Mediating electron transfer from bacteria to a gold electrode via a self-assembled monolayer," Langmuir, 22, 9473 (2006)

\section{CONTACT}

*S. Choi; seokheun.choi@uc.edu 